\title{
- Microtomography-based Inter-Granular Network for the simulation of radionuclide diffusion and sorption in a granitic rock
}

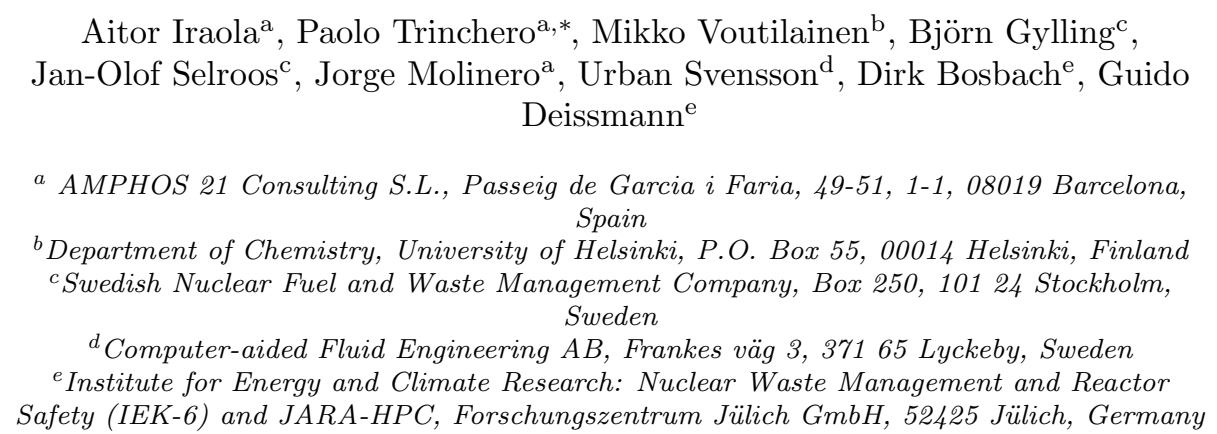

\section{Abstract}

Field investigation studies, conducted in the context of safety analyses of deep geological repositories for nuclear waste, have pointed out that in fractured crystalline rocks sorbing radionuclides can diffuse surprisingly long distances deep into the intact rock matrix; i.e. much longer distances than those predicted by reactive transport models based on a homogeneous description of the properties of the rock matrix. Here, we focus on cesium diffusion and use detailed micro characterisation data, based on micro computed tomography, along with a grain-scale Inter-Granular Network model, to offer a plausible explanation for the anomalously long cesium penetration profiles observed in these in-situ experiments. The sparse distribution of chemically reactive grains (i.e. grains belonging to sorbing mineral phases) is shown to have a strong control on the diffusive patterns of sorbing radionuclides. The computed penetration profiles of cesium agree well with an analytical model based on two parallel diffusive pathways. This agreement, along with visual inspection of the spatial distribution of cesium concentration, indicates that for sorbing radionuclides the medium indeed behaves as a composite system, with most of the mass being retained

\footnotetext{
* Corresponding author

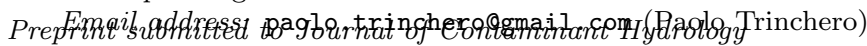


close to the injection boundary and a non-negligible part diffusing faster along preferential diffusive pathways. Keywords: grain-scale model, diffusion experiment, anomalously long cesium

\section{Introduction}

In the last two decades, the description of subsurface flow and transport processes at the pore scale has received increasing attention [1, and references therein]. These modelling efforts have gone hand-in-hand with the increasing availability of laboratory instruments, such as micro computed tomography (CT) scanners, which allow rock samples to be studied down to a resolution of about a micron [e.g. 2, 3, 4].

Besides its academic value, pore scale modelling has been and is being extensively used by, or in the context of, the oil and gas industry, as a cheaper (and, often, more reliable) alternative to laboratory experiments; e.g. to derive continuum-scale properties to be used in reservoir-scale models [e.g. [5] or to study physical and geochemical processes that can influence oil recovery or $\mathrm{CO}_{2}$ storage in applications related to geological carbon sequestration [e.g. 6, 7, 8].

Recently, increasing interest has emerged in using these micro characterisation and modelling techniques for the support of safety analyses of deep geological repositories for nuclear waste. In this context, micro-scale studies are typically applied to fractured crystalline rock formations and focused on assessing the influence of the heterogeneous rock matrix on transport and geochemical processes [9, 10]. Compared to the afore-mentioned applications in the oil and gas industry, one of the main differences here is that diffusion, which is retarded sometimes by sorption mechanisms, is the main (and sometimes only) transport driver in the rock matrix. Moreover, fractured crystalline rocks have usually a very low amount of pore space whose characteristic size is typically below the resolution of micro characterisation techniques. This pore space is typically found in-between grains of different mineral phases [11] and this is why 
these types of micro-scale modelling approaches are denoted here as grain-scale models rather than pore-scale models.

A recent diffusion experiment (LTDE-SD experiment, [12]) carried out in the Äspö Hard Rock Laboratory (HRL) in Sweden has shown that in fractured crystalline rocks strongly sorbing radionuclides can travel surprisingly long distances driven by molecular diffusion. These anomalous penetration profiles are being assessed by an international team of experimentalists and modellers in the framework of the SKB Task Force GWFTS (Groundwater Flow and Transport of Solutes; www.skb.se/taskforce). In this study, we use grain-scale reactive transport models at different levels of detail to give a qualitative explanation to the afore-mentioned experimental results.

More specifically, we apply micro-CT analyses of a crystalline rock sample to provide a description of the inter-granular porosity which is used as topological space for numerical diffusion experiments. A detailed segmentation of the different mineral phases allows chemically reactive grains (i.e. grains where sorbing tracers could potential be sorbed on) to be identified, and sorption sites to be distributed accordingly. The numerical experiments focus on assessing whether the complex porosity network combined with the sparse distribution of sorption sites have a significant impact on the cesium penetration patterns.

\section{X-ray micro-CT characterisation of the rock sample}

The granodiorite sample studied here was taken from the Äspö Hard Rock Laboratory (HRL) is Sweden. The sample (LTDE-SD1) with diameter of $24 \mathrm{~mm}$ and length of $45 \mathrm{~mm}$ was drilled from an exposed fracture surface, which was the object of the LTDE-SD experiment. The rock sample includes a fracture coating with thickness of about $5 \mathrm{~mm}$, an alteration rim, and an underlying less altered rock matrix (Figure 1). The fracture coating consists of calcite and quartz together with minor amounts of chalcopyrite and small crystals of barite and fluorite, and the alteration rim consists mainly of epidote and chlorite [13]. In this work we focus on the less altered rock matrix that is 
fine-grained and according to previous studies [13] consists mainly of quartz (30-35\%), plagioclase (26-33\%), K-feldspar (26-32\%) and biotite (3-7 \%). The samples studied by Widestrand et al. [13] have shown that plagioclase is partly saussuritised and sericitised, and biotite is mostly altered to chlorite. An average porosity of $(0.26 \pm 0.08) \%$ has been determined for the rock matrix and it has been shown that the majority of the porosity is located at the grain boundaries around quartz and feldspar grains, and in biotite grains [13].

The 3D structure of the sample was determined with a SkyScan 1172 microCT scanner which has a conventional X-ray tube with a spot size less than $5 \mu \mathrm{m}$ and a conical X-ray beam. The sample was scanned using a voxel size of $13.58 \mu \mathrm{m}$, an acceleration voltage of $100 \mathrm{kV}$ and a current of $80 \mu \mathrm{A}$. Both aluminum and copper filters were used to treat the energy spectra. During the scan 2040 shadowgrams were taken with a rotation step of $0.10^{\circ}$. The reconstruction of the 3D image was done using a commercial software (NRecon Reconstruction) with beam hardening and ring artefact removal. After 3D reconstruction, 3D realization of Gaussian blur with a radius of 3 voxels was applied to reduce noise from the 3D image and to assist segmentation. Different minerals and the alteration rim were segmented from each other. First the alteration rim was segmented from the rest of the sample by detecting the interface between these two parts using the Carpet algorithm [14]. This was followed by segmentation of different minerals based on their gray value in the 3D image using histogram presentation. Threshold values for segmentation of each mineral were decided such that the thresholds were in between the peaks in the histograms. The rock matrix could be segmented into four mineral phases that include quartz and plagioclase, K-feldspar, mica minerals (biotite and chlorite) and dense accessory minerals. Quartz and plagioclase could not be segmented from each other as their gray values were overlapping due to their similar X-ray attenuation coefficients. Furthermore, distance transform and a watershed algorithm were applied to segment the grain structure of the minerals [15. This was done separately for each mineral phase. The original micro-CT cross section and the images obtained after the segmentation steps are shown in Figure 2. 
The analysis described above was performed for a $1280 \times 1280 \times 1938=$ $3.18 \cdot 10^{9}$ voxels sized representation of the sample with a resolution of $13.58 \mu \mathrm{m}$. The dataset was subsequentially reduced by aggregating voxels up to a resolution scale of $41.19 \mu \mathrm{m}$ that lead to a $422 \times 422 \times 315=5.61 \cdot 10^{7}$ voxels sized representation of the sample after removal of parts including alteration rim. The resulting three-dimensional distribution of mineral grains is shown in Figure 3. The identified mineral phases in the intact rock matrix are quartz and plagioclase, K-feldspar, biotite and accessory minerals, with an abundance of, respectively, $56.1 \%, 42.2 \%, 1.6 \%$ and $0.1 \%$ of the total matrix volume. These values were found to be in fair agreement with the mineral abundances determined previously for Äspö granodiorite [13].

\section{Conceptual model}

The model mimics a typical diffusion experiment, with a cocktail of radionuclides (in this set of calculations a conservative tracer and cesium) that is in contact with one face of the considered rock volume and diffuses into the rock matrix. Previous studies have shown that the properties of alteration rim and fracture coating vary highly within the experimental area of LTDE-SD in Äspö [12. Thus our analysis focuses on the intact rock and the alteration rim was left out of this study (see Figure 3). It is worthwhile noting that in the Äspö LTDE-SD experiment, the diffusion experiment was carried out from an exposed fracture surface but also from a slim hole directly in contact with the intact rock matrix [12. The numerical model presented in this work is qualitatively consistent with this second experimental set-up (i.e. diffusion into the intact rock matrix).

Here, a constant concentration of the conservative tracer and cesium is applied to the bottom boundary of the sample. Both species can penetrate into the sample driven by molecular diffusion while cesium can also sorb onto biotite surfaces. 


\subsection{Construction of an Inter-Granular Network}

A previous study on the distribution of porosity in the LTDE-SD samples carried out using C-14-PMMA autoradiography has shown that a considerable amount of porosity lies within grain boundaries and micro fissures [13]. Hence, it is assumed here that the accessible porosity is mostly constituted by intergranular space (i.e. space between mineral grains), whereas additional intragranular pore space is neglected. There is evidence [4, 16, 17] that, in granitic rocks, dark minerals (e.g. biotite) contain a significant amount of intra-granular pore space. However, in the sample considered in this study there is a low amount of biotite and its mineral grains are small and sparsely distributed (Figure 2 and 3. Thus, it is considered that in this specific rock sample intragranular space has only a minor effect on radionuclide diffusion.

The inter-granular space is conceptualised as a number of rectangular microfractures that are in contact between adjacent mineral grains. Thus, an InterGranular Network (IGN) is built upon a number of Inter-Granular Volumes (IGV's), which are thin rectangular cuboids of dimension $A^{2} \cdot d$, being $A[\mathrm{~m}]$ the voxel size (i.e. $41.19 \mu \mathrm{m})$ and $d[\mathrm{~m}]$ the inter-granular distance. A schematic representation of an IGN is shown in Figure 4. The resulting IGN for the considered rock volume of LTDE-SD1 consists of 23,902,412 IGV's and 59,597,122 connections, is the topological space of the reactive transport problem addressed here.

The distance between adjacent mineral grains (i.e. $d$ in our model) is a highly uncertain parameter. However, a realistic assumption is that the upper threshold for $d$ is below the resolution of the X-ray data (i.e. below $10 \mu \mathrm{m}$ ) and scanning electron microscopy analyses have shown that most of the intergranular distances are close to or below $1 \mu \mathrm{m}$.

With these considerations in mind, a first model, denoted as "Base Case" (BC) model, was built using a constant inter-granular distance of $0.3 \mu \mathrm{m}$, which resulted in a total (bulk) porosity of $0.31 \%$. This value is in agreement with laboratory measurements performed by the water immersion technique in samples drilled in the same rock volume [18]. 
To assess the influence of changes in inter-granular space, with smaller microfractures enhancing diffusive transport and narrowings hindering the transfer of mass, a second set of calculations was considered ("Variant Case" (VC) model). In this case the inter-granular distance was defined using a log-uniform distribution $\left(Y=\log _{10} d\right)$ ranging between $Y=-1.5$ and $Y=0.47$ (i.e. minimum inter-granular distance $d_{\min }=0.03 \mu \mathrm{m}$ and maximum inter-granular distance $\left.d_{\max }=3 \mu \mathrm{m}\right)$. The geometric mean of the inter-granular distance is equal to $0.3 \mu \mathrm{m}$ (i.e. identical to the constant value used in the $\mathrm{BC}$ model) while the arithmetic mean is higher $(0.64 \mu \mathrm{m})$.

\subsection{Set-up of the reactive transport calculations}

The resident water that fills the pore space of the sample is a typical Littorinatype water (e.g. Trinchero et al. [19] and references therein). The composition of the groundwater is provided in Table 1 . The same water, containing a conservative tracer in unitary concentration and cesium in trace concentration $\left(1 \cdot 10^{-8} \mathrm{~mol} / \mathrm{L}\right)$, is applied to the bottom boundary of the domain $\left(\mathrm{z}=\mathrm{z}_{0}\right.$, being $\mathrm{z}_{0}$ the origin of the $\mathrm{z}$ coordinate) using a Dirichlet boundary condition.

It is assumed that at the scale of an IGV, Fick's law is still valid 20]. Molecular diffusion in free water was set to $1 \cdot 10^{-9} \mathrm{~m}^{2} / \mathrm{s}$. In the $\mathrm{BC}$ model local tortuosity, $\tau[-]$, was set to 0.1 resulting in a value of local diffusivity (i.e. diffusivity defined over a support volume of a single IGV) of $D_{p}=1$. $10^{-10} \mathrm{~m}^{2} / \mathrm{s}$. Note that the term "tortuosity" is here used in the same sense of De Marsily [21]; i.e. as the ratio between pore diffusivity and molecular diffusion in free water. This value of local tortuosity has been defined so that the resulting value of macro diffusivity (see section 4.1) is consistent with laboratory experiments carried out in a rock sample taken from the same area of the LTDESD experiment [18].

In the VC model, tortuosity is defined based on the linear expression:

$$
\tau_{i}=\frac{1}{d_{\max }} d_{i}
$$

where subscript $i$ indicates the $\mathrm{i}$-th IGV. 
Eq. (1) assumes that larger inter-granular spaces are completely open $(\tau=$ 1) and that tortuosity decreases linearly with decreasing inter-granular distance. The average value of tortuosity for the VC model is $\bar{\tau}=0.21$, which gives an average local pore diffusivity $\overline{D_{p}}=2.1 \cdot 10^{-10} \mathrm{~m}^{2} / \mathrm{s}$.

Cesium and the other cations sorb onto the available biotite grains (i.e. red grains in Figure 3) via cation exchange, whereas all the other mineral phases are treated as non-sorbing. The sorption model (i.e. cation exchange reactions and related selectivity coefficients - see Table 20 is taken from Kyllönen et al. 22], who assumed three different types of ion exchange sites similar to the model of Bradbury and Baeyens [23] for illite: sites on basal planes (95\% abundance), interlayer sites on crystal edges (frayed-edge sites, FES) (0.02\%) and a third intermediate type site $(5 \%)$.

Kyllönen et al. 22] determined a mass cation exchange capacity for biotite, $C E C^{m}$, equal to $16.3 \mu \mathrm{eq} / \mathrm{g}$. Their experimental analysis was based on samples with a specific surface area of $0.83 \mathrm{~m}^{2} / \mathrm{g}$ (determined with BET analysis), which gives a surface specific $C E C^{s}$ of $19.6 \mu \mathrm{eq} / \mathrm{m}^{2}$. Thus, for each IGV exposed to a biotite grain, a bulk site density is defined as

$$
C E C_{i}^{b}\left[\mathrm{eq} / \mathrm{m}_{\mathrm{b}}^{3}\right]=\mathrm{CEC}^{\mathrm{s}} / \mathrm{d}_{\mathrm{i}}
$$

where superscript $b$ stands for bulk. If none of the two neighboring grains of the IGV are biotite, then the site density is set to zero. For the sake of simplicity, in Eq2 no distinction is made between the case where both neighboring grains are biotite and the case when only one is a chemically reactive grain.

Simulations were carried out using the reactive transport code PFLOTRAN [24, 25].

\section{Results}

Each reactive transport simulation run for $150 \mathrm{~h}$ of simulation time in the supercomputer JUQUEEN of the Jülich Supercomputing Centre [26]. For each 
calculation, a total of 8192 processor cores were used with a wall-clock time of around 12 h. A total of about 200,000 h of supercomputing time were consumed.

\subsection{Conservative tracer}

The penetration of the conservative tracer is analysed by computing the average concentration at given $x-y$ cross section planes and comparing it with the analytical solution for diffusion in a plane sheet [27, 28]:

$$
\frac{C(z, t)}{C_{0}}=1-\frac{4}{\pi} \sum_{n=0}^{\infty} \frac{-1^{n}}{2 n+1} \exp \left(\frac{-D_{a}(2 n+1)^{2} \pi^{2} t}{4 L^{2}}\right) \cos \frac{(2 n+1) \pi(L-z)}{2 L}
$$

where $C_{0}$ is the boundary concentration applied at $z=0$ and $L$ is the total length of the domain. In the analytical model, retention processes are represented using a linear isotherm sorption model. Thus, the apparent diffusivity is here defined as $D_{a}=D_{p}^{*} / R$, being $D_{p}^{*}$ the macro diffusivity (i.e. diffusivity upscaled over a support volume equal to the considered rock sample) and $R$ the retardation factor, which is defined as

$$
R=1+\frac{K_{d} \rho_{s}}{\phi}
$$

being $\rho_{s}\left[\mathrm{~g} / \mathrm{dm}_{\mathrm{b}}^{3}\right]$ and $\phi[-]$ the bulk rock density and porosity, respectively, and $K_{d}[\mathrm{~L} / \mathrm{g}]$ the mass distribution coefficient. For a conservative tracer, $K_{d}=0$; i.e. $R=1$.

The comparison between the numerical results (average concentration computed in 20 equally spaced $x-y$ cross sections) and the analytical solution is shown in Figure 5. The best fit with the analytical solution is obtained using a value of macro diffusivity of $5 \cdot 10^{-11} \mathrm{~m}^{2} / \mathrm{s}$ for the BC model and $2.5 \cdot 10^{-11} \mathrm{~m}^{2} / \mathrm{s}$ for the VC model. For both cases, the agreement between the numerical results and the analytical solution is very good, which indicates that, for conservative diffusive transport, the rock sample behaves as a homogeneous system. Moreover, for both models the value of macro diffusivity is smaller than that of local diffusivity; more specifically, for the $\mathrm{BC}$ model $D_{p}^{*}=D_{p} / 2$ while for the $\mathrm{VC}$ 
model $D_{p}^{*}=\bar{D}_{p} / 8.4$. This decreased macro diffusivity is the result of the intrinsic complexity of the IGN, with solute transport taking place along tortuous connections between IGV's. This effect is further enhanced in the VC model due to the presence of narrowings, which have to be by-passed by solute mass, thus increasing the overall tortuosity of the system.

4.2. Cesium

Snapshots of tracer, cesium and sorbed cesium concentration computed at $10 \mathrm{~h}$ and $150 \mathrm{~h}$ with the $\mathrm{BC}$ and VC models are shown in Figures 6 and 7 respectively. As already observed in the previous section, it can be noticed that, at time $150 \mathrm{~h}$, the conservative tracer has traveled a longer distance in the $\mathrm{BC}$ model than in the $\mathrm{VC}$ model. By a simple visual inspection, it is evident that cesium penetrates at much slower rates than tracer as a result of sorption processes. Consistently with the underlying conceptual model, cesium is sorbed in few IGV's that are neighboring of biotite grains.

In Figure 8, a cross section located at $z=2.59 \cdot 10^{-3} \mathrm{~m}$ is used to show snapshots of conservative tracer, aqueous cesium and sorbed cesium concentration computed with the VC model at $150 \mathrm{~h}$. A smooth spatial distribution is observed for the conservative tracer. The modest concentration gradients are the result of the internal variability of diffusive pathways, which in the VC model is further enhanced by the variable distribution of inter-granular space and tortuosity. A completely different behavior is observed for cesium, which shows strong gradients between spots of high concentration and regions with very low concentration. The effect of the sparsely distributed exchange sites is evident by visually comparing the distribution of aqueous cesium and sorbed cesium concentration. Cesium is sorbed in very few exposed IGV's (i.e. the inter-granular volumes that are neighbors of biotite grains). However, these exposed IGV's behave as sinks of radionuclides and their influence on the transport of aqueous cesium extends far beyond the location of biotite grains, keeping cesium aqueous concentration at low levels also in surrounding areas. Furthermore, these cesium concentration patterns are similar to the ones observed from similar diffusion 
experiments and analysed using autoradiographic techniques [29, 30, 12].

The penetration profiles of aqueous cesium obtained with the two models at time $150 \mathrm{~h}$ are shown in Figure 9. The penetration depth is lower in the VC model than in the BC model due to its lower (estimated) macro diffusivity (section 4.1). These profiles are compared with the analytical solutions (Eq. 3) computed with the macro diffusivity values estimated in section 4.1 and with $R=25$. The retardation factor was calibrated to match the first part of each profile. An additional reactive transport calculation was carried out with a homogeneous 1D system based on the bulk parameters of the BC model. In this simulation, which includes the geochemical reactions described in section 3.2 the biotite volume fraction was set to $1.6 \%$ (i.e. same abundance as in the LTDE-SD1 sample), which resulted in a total amount of sites $C E C^{b}=0.77$ $\mathrm{eq} / \mathrm{m}_{\mathrm{b}}^{3}$. The comparison of both simulations points out that, for a sorbing tracer, the considered rock sample is not well described by an equivalent homogeneous model. In particular, mineralogical heterogeneity leads to much longer penetration depths.

In in-situ diffusion experiments, the rock surrounding the experimental area is typically over-cored after termination of the experiment and a number of small core samples are drilled from the over-cored rock volume. A post-mortem analysis is then carried out by sawing the samples into slices. Each slice is then analysed by means of direct activity measurements, which provide an estimation of the total radionuclide mass contained in the considered slice. To mimic this experimental approach, penetration profiles of total cesium mass computed at $150 \mathrm{~h}$ for the two IGN models, the analytical solutions and the homogeneous simulation are shown in Figure 10. Mass is normalised by the total mass in the first slice (i.e. the slice located closest to the exposed face of the rock sample). As expected, given the underlying assumption of linearity, the analytical results are the same already observed for aqueous concentration (Figure 9). Slight deviations from the profiles of aqueous concentrations are observed for the numerical calculations (including the homogeneous 1D simulation). These deviations are the result of the non-linear sorption model, whose exchange sites might become 
depleted, particularly close to the exposed boundary. As in the case of aqueous concentrations, none of the homogeneous models is able to capture the long penetration tails observed in the two IGN calculations.

The visual inspection of the spatial distribution of cesium (Figure 8) has already pointed out that the medium behaves as a composite system, with most of the mass being strongly retained close to the biotite grains and a nonnegligible part of it which diffuses along preferential diffusive pathways. A similar dichotomy is observed when analysing flow through unsaturated waste rock piles, with most of the infiltrating water flowing slowly through the matrix and a small part of it being quickly channeled along macropores ([e.g. 31]). Similarly to the approach employed by Trinchero et al. 32 to simulate flow in a mining waste rock pile, here we use an alternative analytical model based on two parallel diffusive pathways:

$$
C(z, t)=\alpha C_{\text {slow }}(z, t)+(1-\alpha) C_{\text {fast }}(z, t)
$$

A similar approach was also used by Cvetkovic 33 to evaluate the results of the LTDE-SD experiment.

In Eq.(5), both $C_{\text {slow }}$ and $C_{\text {fast }}$ are obtained from Eq.(3) using the values of macro diffusivity estimated for the $\mathrm{BC}$ and $\mathrm{VC}$ models but with different retardation factors. The retardation of the "slow" paths $\left(R_{\text {slow }}\right)$ is set to 25 in both models; i.e. the same value used in the homogeneous analytical model to fit the first part of each computed profile. The retardation values of the "fast" paths (i.e. $R_{\text {fast }}$ ) and $\alpha$ are the calibration parameters. The best fit with the IGN calculations (Figure 11) is obtained by setting $\alpha=0.9$, for both models, and $R_{\text {fast }}$ to 5.8 and 4.1 for the $\mathrm{BC}$ and $\mathrm{VC}$ model, respectively. The slightly lower retardation factor of the $\mathrm{VC}$ model is related to the fact that the average inter-granular distance of the $\mathrm{VC}$ model is larger than the (constant) value used in the BC model, which results in a lower average density of exchange sites (Eq2). The overall good agreement between the two-parallel-pathway models and the IGN results points out that the system indeed behaves as a 
composite medium with approximately $90 \%$ of the mass being retained in or nearby strongly sorbing area, and the remaining $10 \%$ of the mass that diffuses along pathways that exhibit less retention potential.

It is worthwhile stressing that this composite behavior depends in turn on the ratio between the characteristic length of the problem and the average distance between chemically reactive grains. In the considered problem, the characteristic distance sampled by cesium is $L \sim 1 \cdot 10^{-2} \mathrm{~m}$ whereas the average distance between grains is $d_{\text {grain }} \sim 3 \cdot 10^{-3} \mathrm{~m}$, which gives a ratio $L / d_{\text {grain }} \sim 3.3$. For larger ratios, i.e. when cesium samples a larger part of the matrix, deviations from the homogeneous behavior are expected to be less significant. A qualitatively similar behavior is observed when interpreting drawdown data from a pumping test, with the heterogeneous aquifer acting as an equivalent homogeneous system at late times; i.e. when the cone of depression has sampled a large portion of the aquifer [34, 35].

\section{Discussion and conclusions}

The SKB Task Force GWFTS is an international forum supporting the Äspö Hard Rock Laboratory activities. Within this forum, different modelling groups interact in the area of conceptual and numerical modelling of groundwater flow and solute transport in fractured rock, with special emphasis on specific in-situ experiments carried out in the HRL facility.

Recently, a new task (Task 9B) has been established, which aims at reinterpreting the results of the LTDE-SD experiments. As explained in the memorandum of Task 9B, "[prior to the start of the Task 9B activities] predictive modelling was made for the tracer penetration profiles, based on laboratory data, and [...] inverse modelling was made based on the obtained in-situ data. Regardless of the chosen parameters, the discrepancies between the modelled and observed profiles were more pronounced than the similarities". Of special concern for Task 9B are the anomalously long penetration distances observed for sorbing and strongly sorbing radionuclides Nilsson et al. [12]. 
Here, we have used a detailed micro-characterisation study of a rock sample, taken in the area of the experiment, along with grain-scale reactive transport models to offer a qualitative interpretation for these anomalously long penetration profiles. The results of the calculations have pointed out that the sparse availability of exchange sites has a strong influence on the observed cesium penetration pattern. The profiles of cesium penetration agree well with a simplified lumped model based on two parallel diffusive pathways. This match points out that, for sorbing radionuclides, the considered rock volume behaves as a composite medium, with most of the mass (around 90\%) being retained close to the injection boundary and a non-negligible part of the solute (around 10\%) moving faster along preferential diffusive pathways. A qualitatively similar behavior is observed in different cores drilled in the experimental area of LTDE-SD and a similar parallel pathways model was successfully used by Cvetkovic 33. to provide a quantitative explanation to these observed anomalous penetration profiles.

Other main conclusions of the work are:

- the penetration profiles of the conservative tracer resemble those obtained from homogeneous models. The intrinsic complexity of the IGN results in a macro diffusivity that is lower than the values set at the scale of a single IGV.

- when internal variability of inter-granular space is taken into account, macro diffusivity is further decreased due to the effect of narrowings, which have to be by-passed by diffusing solutes.

- active grains behave as sinks for aqueous radionuclides and their influence extends far beyond the location of the grains itself.

It is worthwhile noting that the conclusions obtained from this work do not exclude other (additive) effects that could be important over the (larger) scale of the LTDE-SD experiment. Features that might also contribute to anomalous 
penetration profiles (also for conservative tracers) are cm-scale fractures and skin effects due to local stress redistribution [36].

Acknowledgements AI, PT, MV, JM and US thank the Swedish Nuclear Fuel and Waste Management Company (SKB) for the financial support. The authors also thank the PFLOTRAN development group for their help during the project. The authors gratefully acknowledge the computing time granted by the JARA-HPC Vergabegremium and provided on the JARA-HPC Partition part of the supercomputer JUQUEEN at Forschungszentrum Jülich. This work has benefited from insightful comments by Bill Lanyon and Josep Soler, respectively chairman and evaluator of Task 9 of the SKB Task Force GWFTS.

\section{References}

\section{References}

[1] S. Molins, Reactive interfaces in direct numerical simulation of pore scale processes, Reviews in Mineralogy and Geochemistry 80 (2015) 461-481.

[2] M. J. Blunt, B. Bijeljic, H. Dong, O. Gharbi, S. Iglauer, P. Mostaghimi, A. Paluszny, C. Pentland, Pore-scale imaging and modelling, Advances in Water Resources 51 (2013) 197-216.

[3] F. Fusseis, X. Xiao, C. Schrank, F. De Carlo, A brief guide to synchrotron radiation-based microtomography in (structural) geology and rock mechanics, Journal of Structural Geology 65 (2014) 1-16.

[4] M. Voutilainen, M. Siitari-Kauppi, P. Sardini, A. Lindberg, J. Timonen, Porespace characterization of an altered tonalite by x-ray computed microtomography and the 14c-labeled-polymethylmethacrylate method, Journal of Geophysical Research 117 (2012). B01201.

[5] P.-E. Øren, S. Bakke, Reconstruction of Berea sandstone and pore-scale modelling of wettability effects, Journal of Petroleum Science and Engineering 39 (2003) 177-199. 
[6] M. Andrew, B. Bijeljic, M. J. Blunt, Pore-scale imaging of geological carbon dioxide storage under in situ conditions, Geophysical Research Letters 40 (2013) 3915-3918.

[7] S. Molins, D. Trebotich, L. Yang, J. B. Ajo-Franklin, T. J. Ligocki, C. Shen, C. I. Steefel, Pore-scale controls on calcite dissolution rates from flowthrough laboratory and numerical experiments, Environmental Science \& Technology 48 (2014) 7453-7460.

[8] X. Huang, K. W. Bandilla, M. A. Celia, Multi-physics pore-network modeling of two-phase shale matrix flows, Transport in Porous Media 111 (2016) $123-141$.

[9] M. Voutilainen, P. Sardini, M. Siitari-Kauppi, P. Kekäläinen, V. Aho, M. Myllys, J. Timonen, Diffusion of tracer in altered tonalite: experiments and simulations with heterogeneous distribution of porosity, Transport in Porous Media 96 (2013) 319-336.

[10] P. Trinchero, J. Molinero, G. Deissmann, U. Svensson, B. Gylling, H. Ebrahimi, G. Hammond, D. Bosbach, I. Puigdomenech, Implications of grain-scale mineralogical heterogeneity for radionuclide transport in fractured media, Transport in Porous Media 1 (2017) 73-90.

[11] M. Voutilainen, J. Ikonen, J. Sammaljärvi, J. Kuva, A. Lindberg, M. SiitariKauppi, L. Koskinen, Through diffusion study on Olkiluoto veined gneiss and pegmatitic granite from a structural perspective, MRS Advances 1 (2016) 4041-4046.

[12] K. Nilsson, J. Byegård, E. Selnert, H. Widestrand, S. Höglund, E. Gustafsson, Äspö Hard Rock Laboratory. Long Term Sorption Diffusion Experiment (LTDE-SD). Results from rock sample analyses and modelling., Technical Report R-10-68, Svensk Kärnbränslehantering AB, Stockholm, Sweden, 2010. 
[13] H. Widestrand, J. Byegård, E. Selnert, M. Skålberg, S. Höglund, E. Gustafsson, Long Term Sorption Diffusion Experiment (LTDESD). Supporting laboratory program - Sorption diffusion experiments and rock material characterisation, Technical Report R-10-66, Svensk Kärnbränslehantering AB, Stockholm, Sweden, 2010.

[14] T. Turpeinen, M. Myllys, P. Kekäläinen, J. Timonen, Interface detection using a quenched-noise version of the Edwards-Wilkinson equation, IEEE Transactions on Image Processing 24 (2015) 5696-5705.

[15] P. Sardini, S. Sammartino, E. Tévissen, An image analysis contribution to the study of transport properties of low-permeability crystalline rocks, Computers \& geosciences 27 (2001) 1051-1059.

[16] P. Sardini, M. Siitari-Kauppi, D. Beaufort, K.-H. Hellmuth, On the connected porosity of mineral aggregates in crystalline rocks, American Mineralogist 91 (2006) 1069-1080.

[17] A. Mazurier, P. Sardini, A. M. Rossi, R. C. Graham, K.-H. Hellmuth, J.-C. Parneix, M. Siitari-Kauppi, M. Voutilainen, L. Caner, Development of a fracture network in crystalline rocks during weathering: Study of Bishop Creek chronosequence using X-ray computed tomography and 14C-PMMA impregnation method, Geological Society of America Bulletin 128 (2016) 1423-1438.

[18] P. Vilks, N. Miller, F. Stanchell, Laboratory program supporting SKBs long term diffusion experiment, Ontario Power Generation, Nuclear Waste Management Division Supporting Technical Report (2005).

[19] P. Trinchero, I. Puigdomenech, J. Molinero, H. Ebrahimi, B. Gylling, U. Svensson, D. Bosbach, G. Deissmann, Continuum-based DFN-consistent numerical framework for the simulation of oxygen infiltration into fractured crystalline rocks, Journal of Contaminant Hydrology 200 (2017) 60-69. 
[20] J.-M. Philibert, Atom movements-Diffusion and mass transport in solids, EDP Sciences, 2012.

[21] G. De Marsily, Quantitative hydrogeology, 1986.

[22] J. Kyllönen, M. Hakanen, A. Lindberg, R. Harjula, M. Vehkamäki, J. Lehto, Modeling of cesium sorption on biotite using cation exchange selectivity coefficients, Radiochimica Acta 102 (2014) 919-929.

[23] M. H. Bradbury, B. Baeyens, A generalised sorption model for the concentration dependent uptake of caesium by argillaceous rocks, Journal of Contaminant Hydrology 42 (2000) 141-163.

[24] P. Lichtner, G. E. Hammond, C. Lu, S. Karra, G. Bisht, B. Andre, R. Mills, J. Kumar, PFLOTRAN User Manual, Technical Report, 2013.

[25] G. Hammond, P. Lichtner, R. Mills, Evaluating the performance of parallel subsurface simulators: An illustrative example with PFLOTRAN, Water Resources Research 50 (2014) 208-228.

[26] M. Stephan, J. Docter, JUQUEEN: IBM Blue Gene/Q Supercomputer System at the Jülich Supercomputing Centre, Journal of large-scale research facilities JLSRF 1 (2015).

[27] H. Carslaw, J. Jaeger, Conduction of heat in solids, Oxford: Clarendon Press, 1959, 2nd ed. 1 (1959).

[28] J. Crank, The mathematics of diffusion, Oxford university press, 1979.

[29] H. Johansson, M. Siitari-Kauppi, M. Skålberg, E.-L. Tullborg, Diffusion pathways in crystalline rock - examples from Äspö-diorite and fine-grained granite, Journal of Contaminant Hydrology 35 (1998) 41-53.

[30] L. Jokelainen, T. Meski, A. Lindberg, J. Soler, M. Siitari-Kauppi, A. Martin, J. Eikenberg, The determination of ${ }^{134} \mathrm{Cs}$ and ${ }^{22} \mathrm{Na}$ diffusion profiles in granodiorite using gamma spectroscopy, Journal of Radioanalytical and Nuclear Chemistry 295 (2013) 2153-2161. 
[31] C. Nichol, L. Smith, R. Beckie, Field-scale experiments of unsaturated flow and solute transport in a heterogeneous porous medium, Water Resources Research 41 (2005).

[32] P. Trinchero, R. Beckie, X. Sanchez-Vila, C. Nichol, Assessing preferential flow through an unsaturated waste rock pile using spectral analysis, Water Resources Research 47 (2011) W07532.

[33] V. Cvetkovic, Evaluation of LTDE results, unpublished report (2016).

[34] X. Sanchez-Vila, P. M. Meier, J. Carrera, Pumping tests in heterogeneous aquifers: An analytical study of what can be obtained from their interpretation using jacob's method, Water Resources Research 35 (1999) 943-952.

[35] M. T. Demir, N. K. Copty, P. Trinchero, X. Sanchez-Vila, Bayesian estimation of the transmissivity spatial structure from pumping test data, Advances in Water Resources 104 (2017) 174-182.

[36] P. Vilks, J. Cramer, M. Jensen, N. Miller, H. Miller, F. Stanchell, In situ diffusion experiment in granite: phase i, Journal of Contaminant Hydrology 61 (2003) 191-202. 
Table 1: Chemical composition of the groundwater used in the reactive transport calculations.

\begin{tabular}{ll}
\hline Total concentration $(\mathrm{mol} / \mathrm{L})$ & \\
\hline $\mathrm{Na}^{+}$ & $3.3 \times 10^{-2}$ \\
$\mathrm{~K}^{+}$ & $6.1 \times 10^{-4}$ \\
$\mathrm{Ca}^{2+}$ & $9.5 \times 10^{-4}$ \\
$\mathrm{Cl}^{-}$ & $3.1 \times 10^{-2}$ \\
$\mathrm{SO}_{4}^{2-}$ & $2.1 \times 10^{-3}$
\end{tabular}


Table 2: Cation exchange reactions and selectivity coefficients (Gaines-Thomas convention)

$22]$.

\begin{tabular}{lr}
\hline Reaction & Selectivity coefficients $(\log K)$ \\
\hline Planar sites & \\
$\mathrm{X}^{-}+\mathrm{Na}^{+} \leftrightarrow \mathrm{NaX}$ & 0.0 \\
$\mathrm{X}^{-}+\mathrm{K}^{+} \leftrightarrow \mathrm{KX}$ & 2.3 \\
$\mathrm{X}^{-}+\mathrm{Cs}^{+} \leftrightarrow \mathrm{CsX}$ & 2.0
\end{tabular}

Intermediate sites

$\begin{array}{lr}\mathrm{X}_{\text {int }}^{-}+\mathrm{Na}^{+} \leftrightarrow \mathrm{NaX}_{\text {int }} & 0.0 \\ \mathrm{X}_{\text {int }}^{-}+\mathrm{K}^{+} \leftrightarrow \mathrm{KX}_{\text {int }} & 2.3 \\ \mathrm{X}_{\text {int }}^{-}+\mathrm{Cs}^{+} \leftrightarrow \mathrm{CsX}_{\text {int }} & 4.5\end{array}$

Frayed-edge sites

$\mathrm{X}_{\mathrm{FES}}^{-}+\mathrm{Na}^{+} \leftrightarrow \mathrm{NaX}_{\mathrm{FES}}$

$\mathrm{X}_{\mathrm{FES}}^{-}+\mathrm{K}^{+} \leftrightarrow \mathrm{KX}_{\mathrm{FES}}$

$\mathrm{X}_{\mathrm{FES}}^{-}+\mathrm{Cs}^{+} \leftrightarrow \mathrm{CsX}_{\mathrm{FES}}$ 

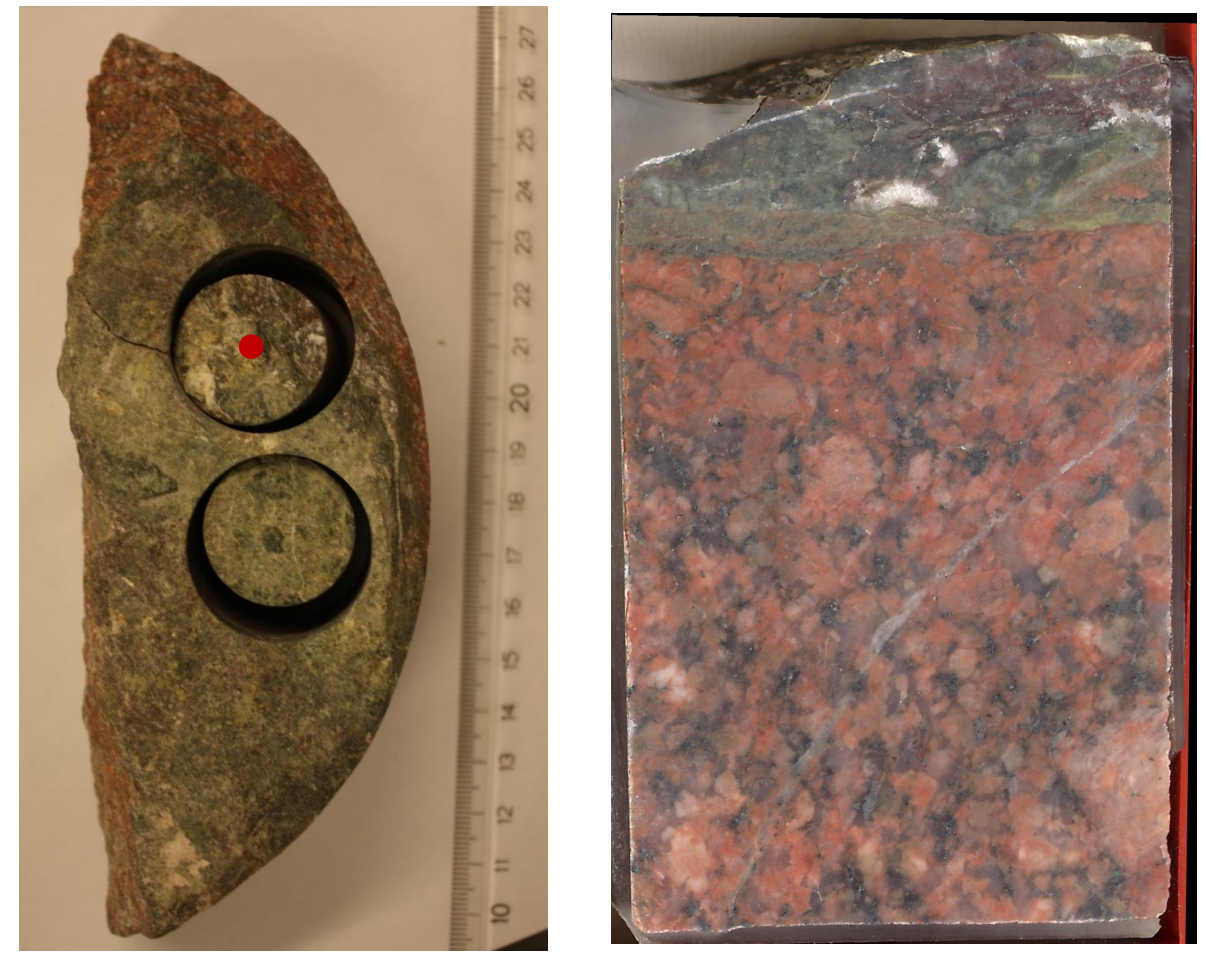

Figure 1: (left) The LTDE-SD samples from the Äspö HRL (Sweden) and (right) cross section of sample LTDE-SD1, which is the object of this study (sample LTDE-SD1 is also indicated with a red dot in the picture to the left). 


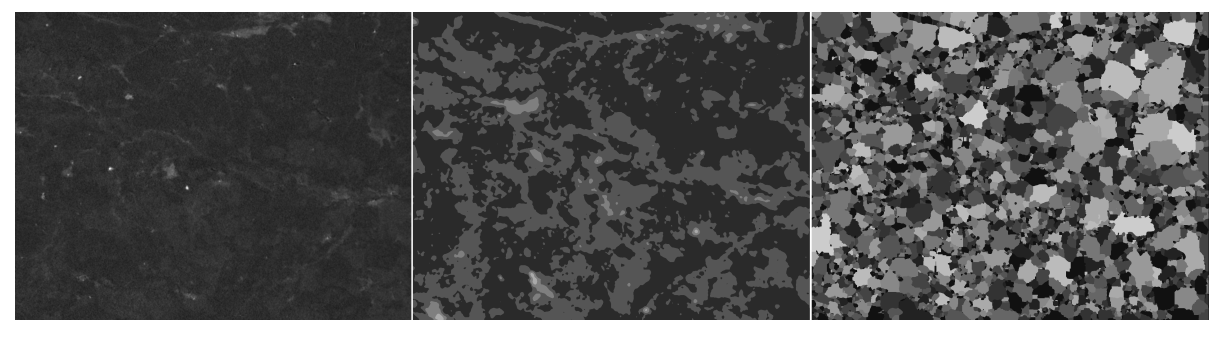

Figure 2: Cross sections of the LTDE-SD1 sample showing original micro-CT (left), map of segmented mineral phases (center) and map of segmented mineral grains (right). In the map of segmented mineral phases quartz and plagioclase (dark gray), K-feldspar (gray) and biotite (light gray) are shown.

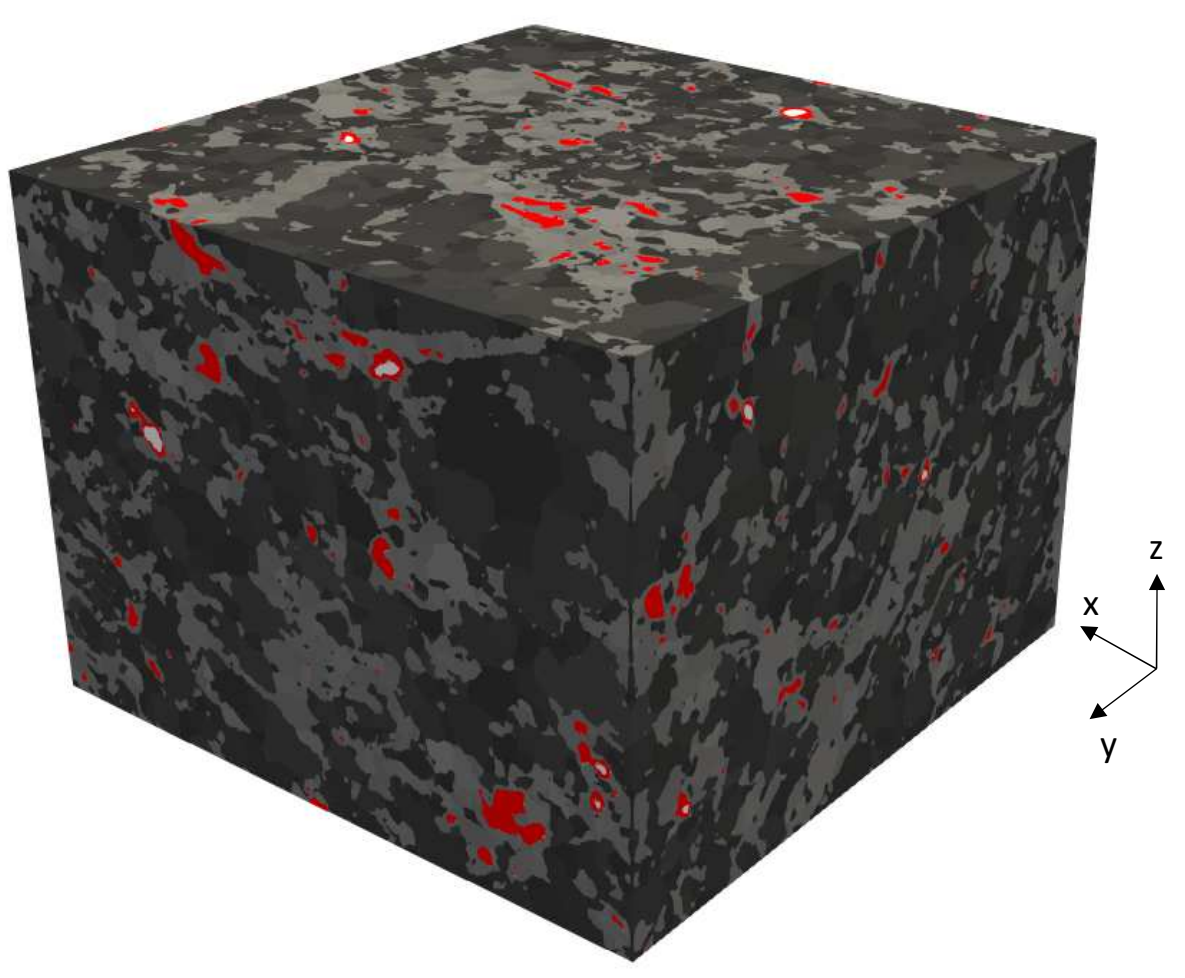

Figure 3: Three-dimensional visualisation of the LTDE-SD1 sample after mineral segmentation. Only the part of the sample considered in the calculations is shown $\left(1.7 \cdot 10^{-2} \mathrm{~m} \mathrm{x}\right.$ $\left.1.7 \cdot 10^{-2} \mathrm{~m} \mathrm{x} 1.3 \cdot 10^{-2} \mathrm{~m}\right)$. Biotite grains are displayed in red. 


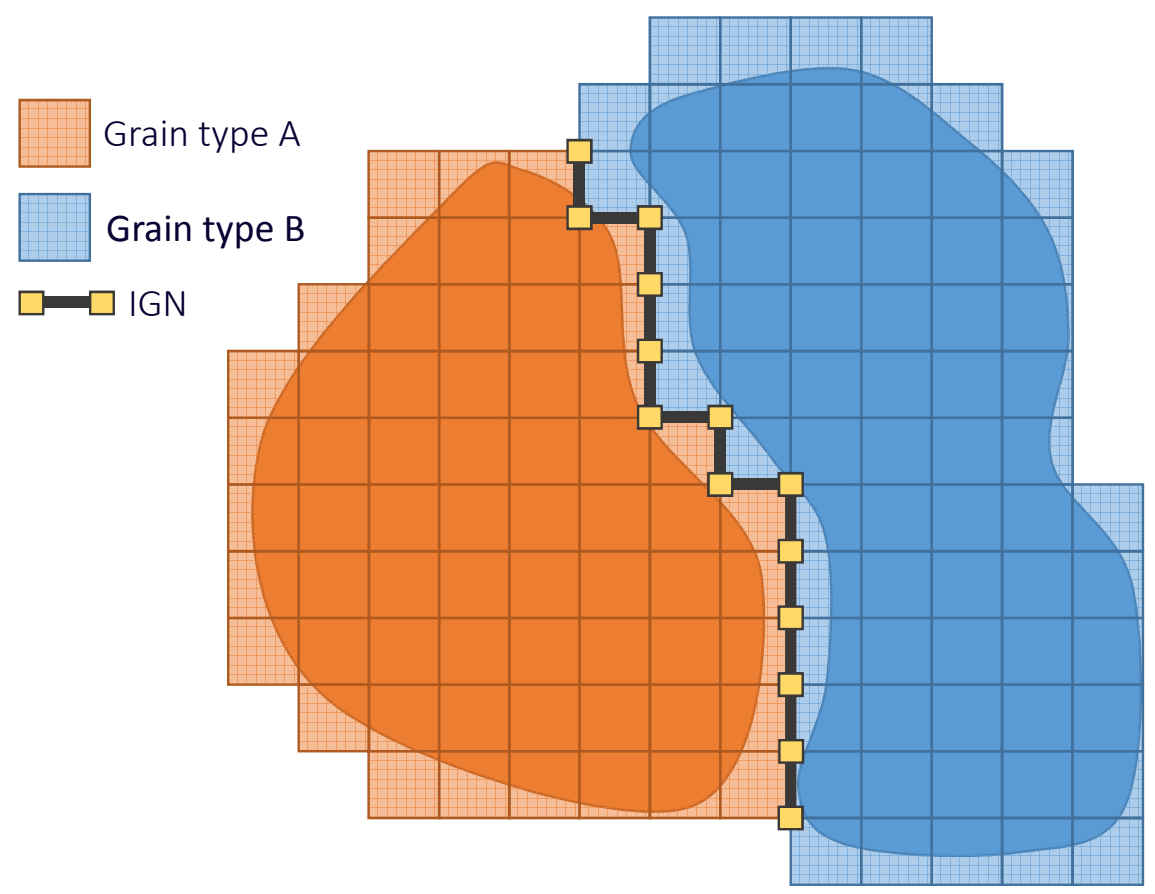

Figure 4: Inter-Granular Network (IGN) formed by surface voxels of segmented mineral grains (A and B). 


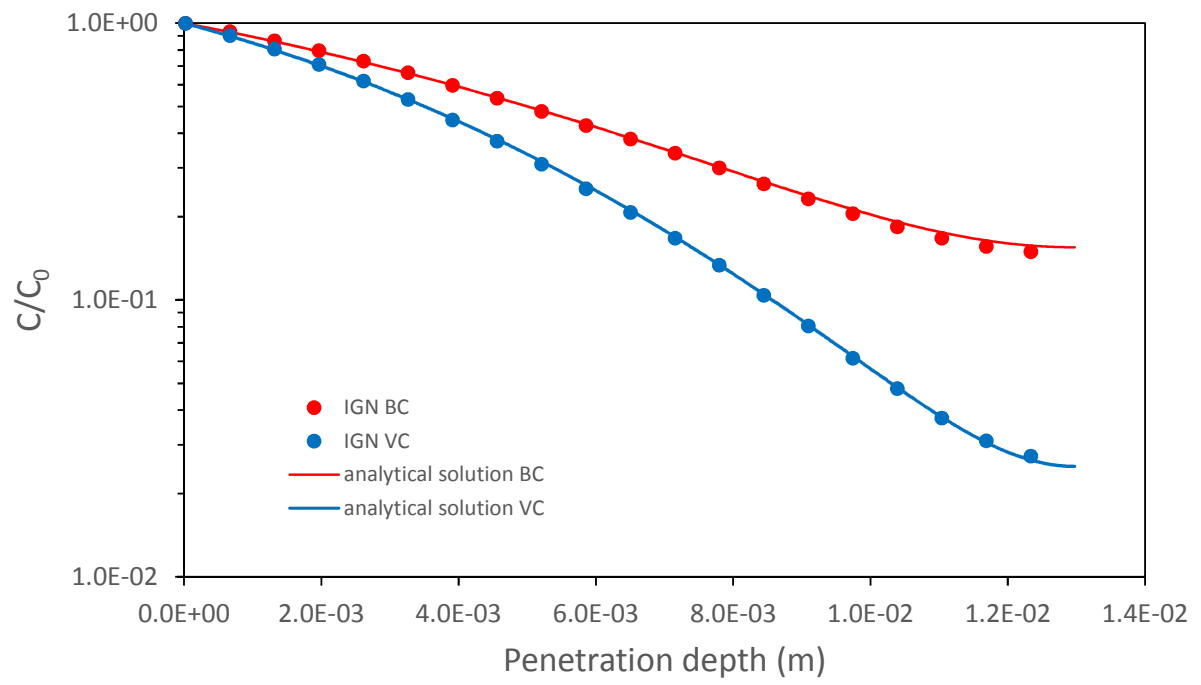

Figure 5: Penetration profiles for the conservative tracer computed at $150 \mathrm{~h}$ for the $\mathrm{BC}$ (red dots) and VC (blue dots) models. Both results can be fitted with the analytical solution for diffusion in a plane sheet (Eq. 3 and continuous lines). The best fit with the analytical solution is obtained using a value of macro diffusivity of $5 \cdot 10^{-11} \mathrm{~m}^{2} / \mathrm{s}$ for the BC model and $2.5 \cdot 10^{-11} \mathrm{~m}^{2} / \mathrm{s}$ for the VC model. 


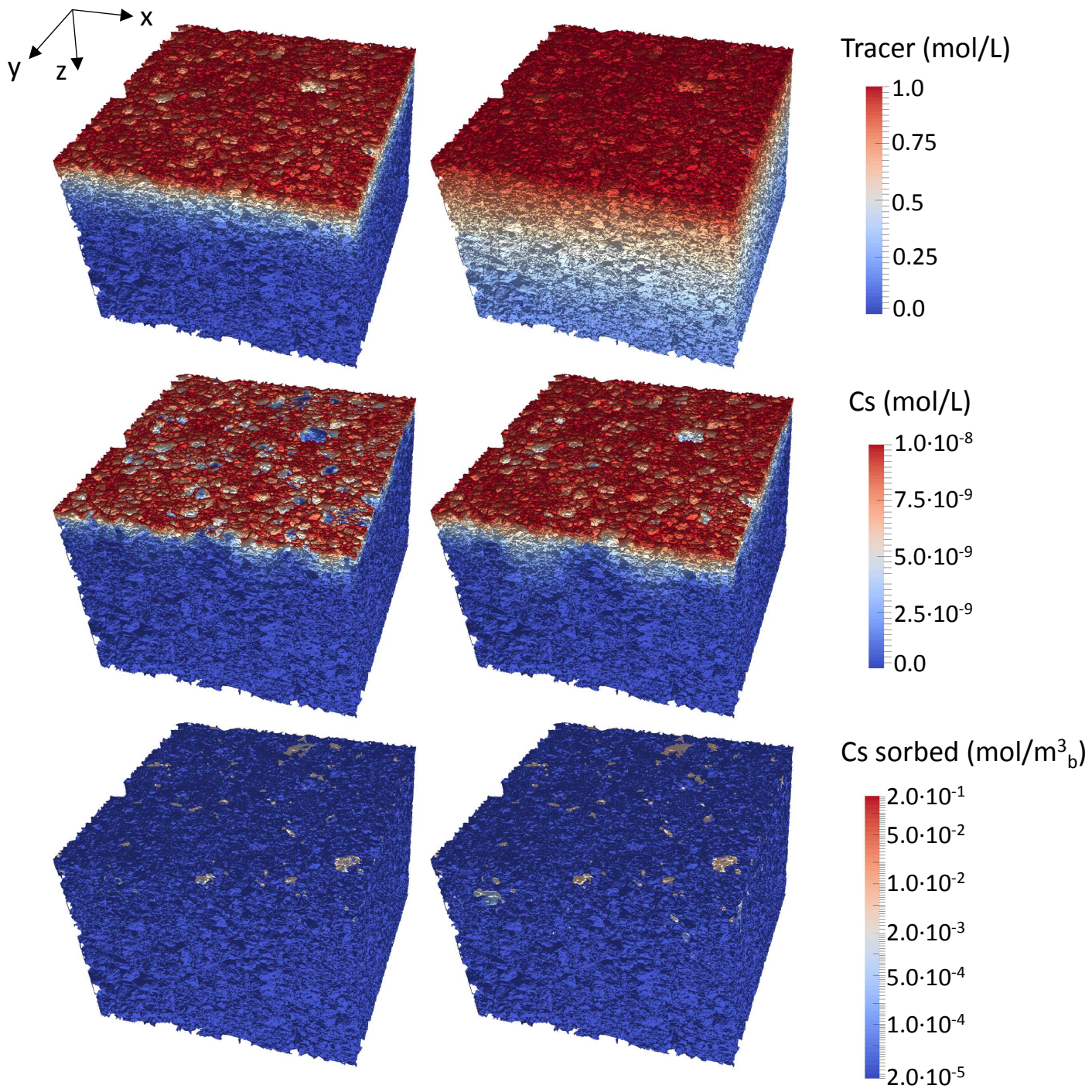

Figure 6: Snapshots of (top) conservative tracer, (middle) aqueous cesium and (bottom) cesium sorbed computed at (left) $10 \mathrm{~h}$ and (right) $150 \mathrm{~h}$ with the BC model. 


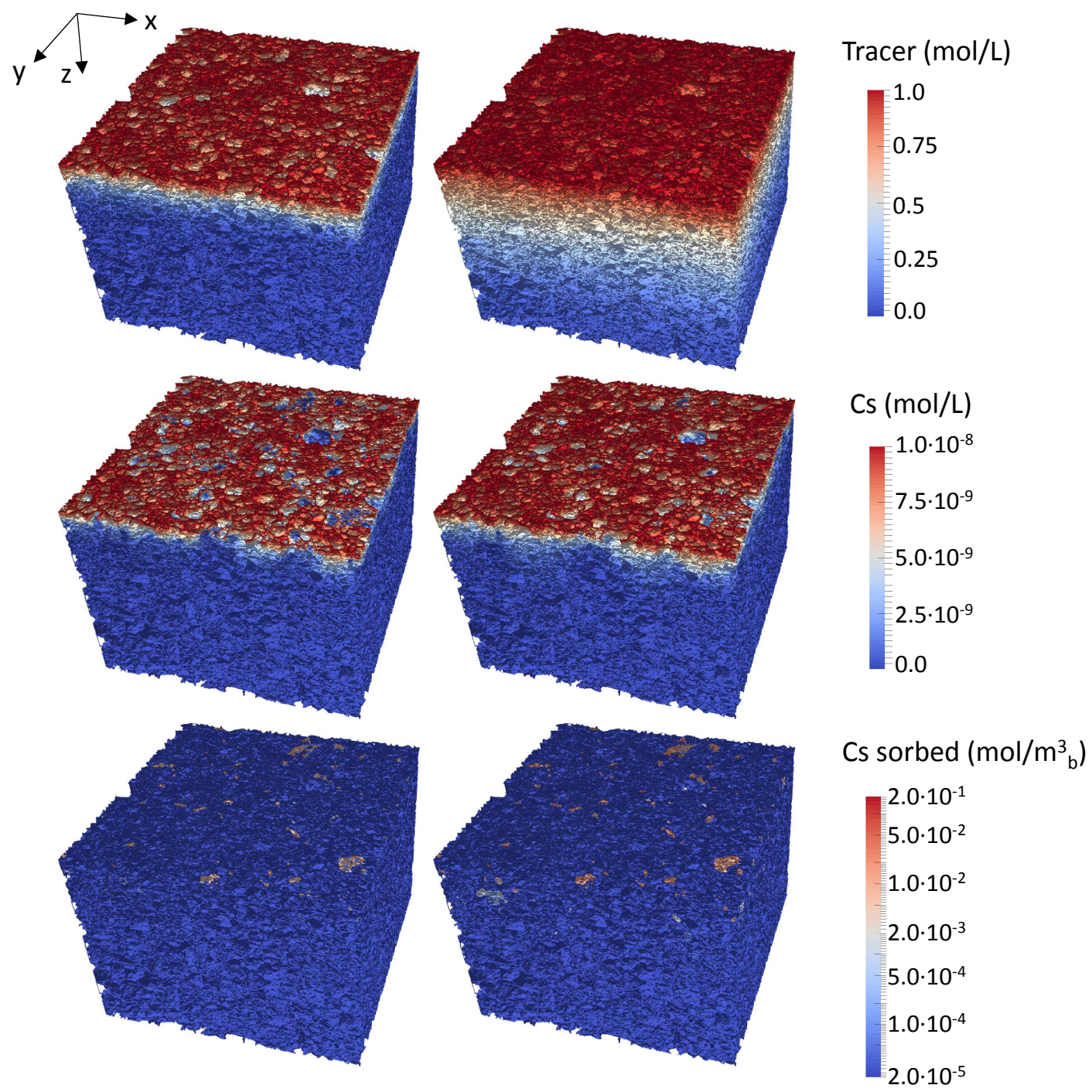

Figure 7: Snapshots of (top) conservative tracer, (middle) aqueous cesium and (bottom) cesium sorbed computed at (left) $10 \mathrm{~h}$ and (right) $150 \mathrm{~h}$ with the $\mathrm{VC}$ model. 


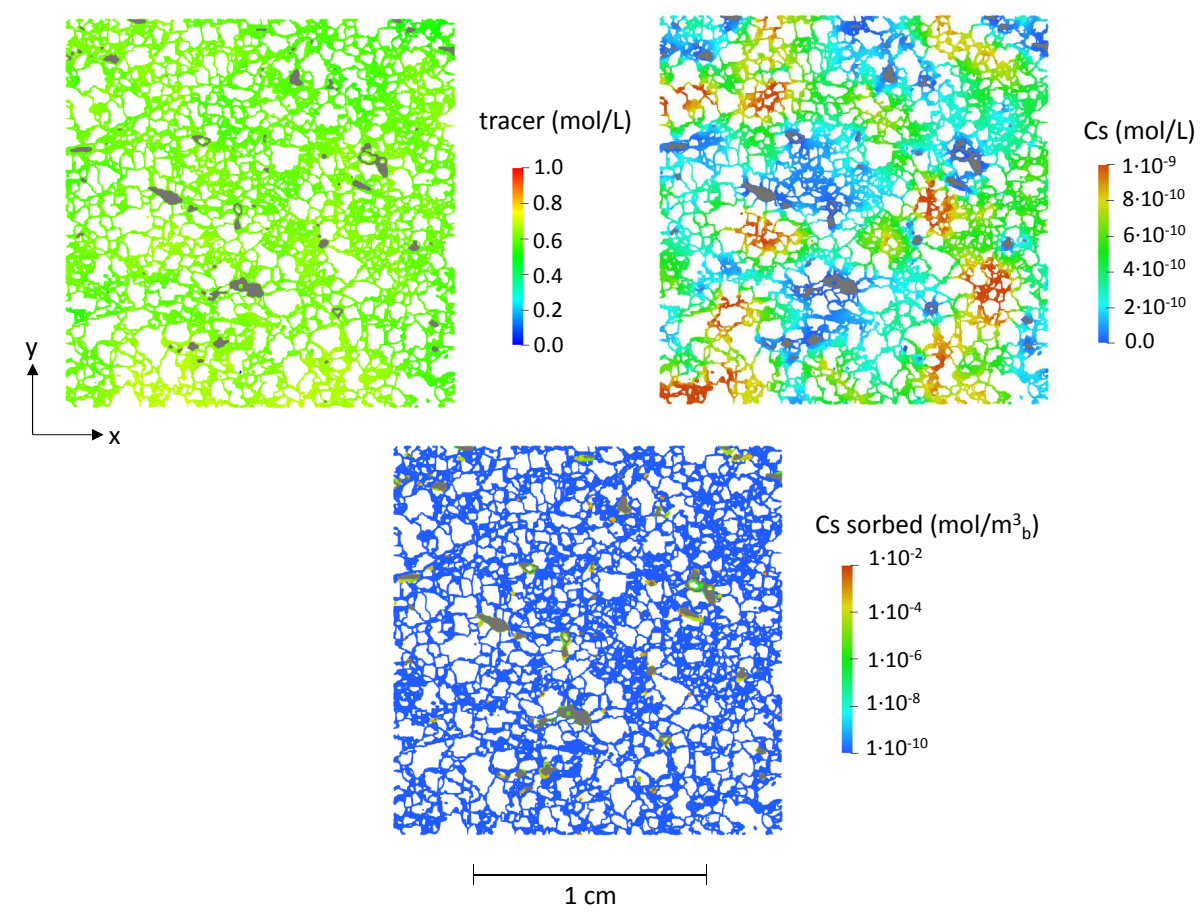

Figure 8: Distribution of (top-left) conservative tracer, (top-right) aqueous cesium and (bottom) sorbed cesium concentration computed at $150 \mathrm{~h}$ with the $\mathrm{VC}$ model. Results are shown in a cross section taken at $z=2.59 \cdot 10^{-3} \mathrm{~m}$. Gray shaded areas represent the biotite grains. 


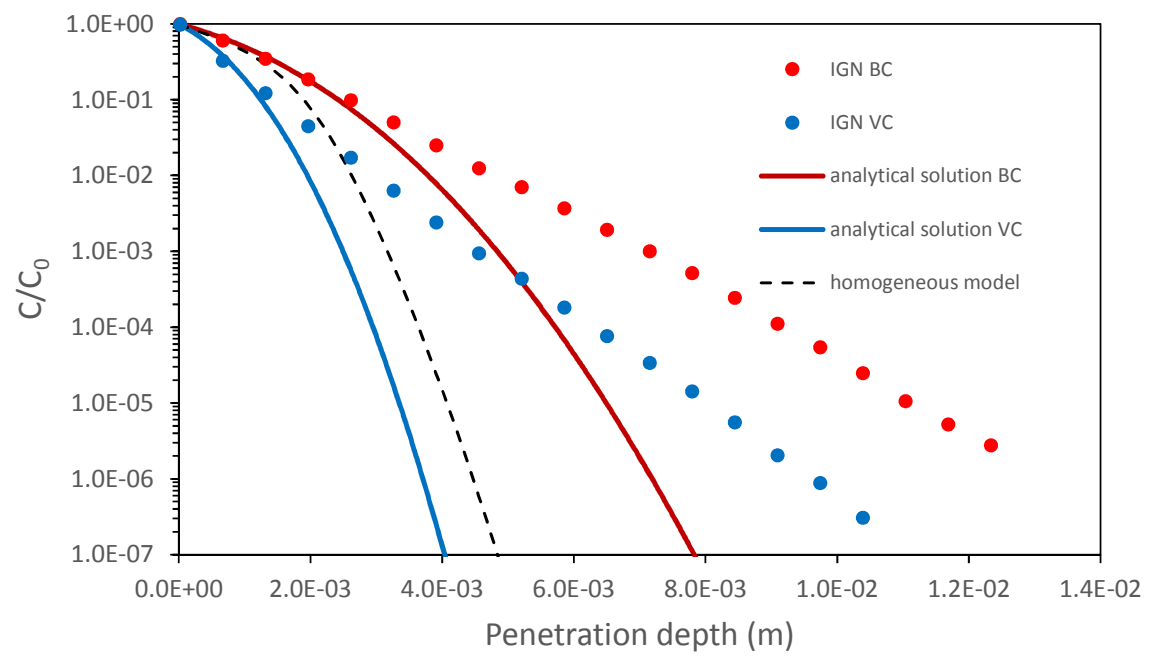

Figure 9: Penetration profiles for cesium (aqueous cesium concentration) computed at $150 \mathrm{~h}$ for the BC (red dots) and VC (blue dots) models. The analytical solutions used to fit the numerical results are shown with continuous lines whereas the dashed line shows the results of a numerical calculation based upon a homogeneous distribution of the available exchange sites. 


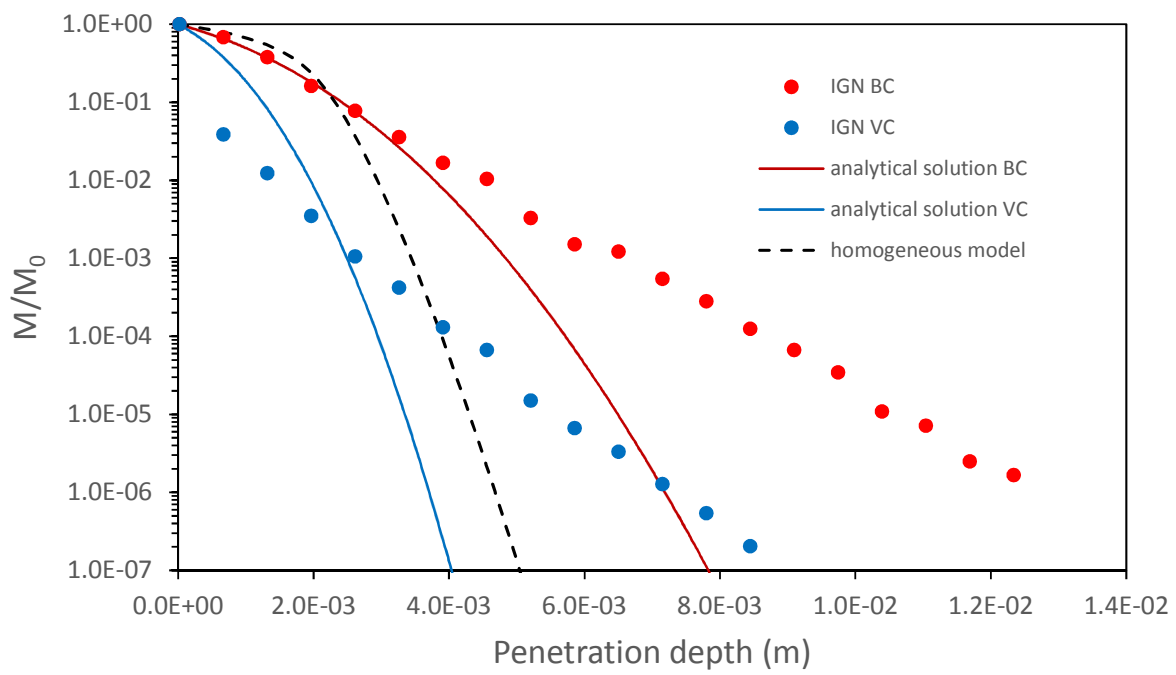

Figure 10: Total mass of cesium computed at $150 \mathrm{~h}$ for the BC (red dots) and VC (blue dots) models. The analytical solutions used to fit the numerical results are shown with continuous lines whereas the dashed line shows the results of a numerical calculation based upon a homogeneous distribution of the available exchange sites. Mass is normalised by the total mass close to the injection boundary. 


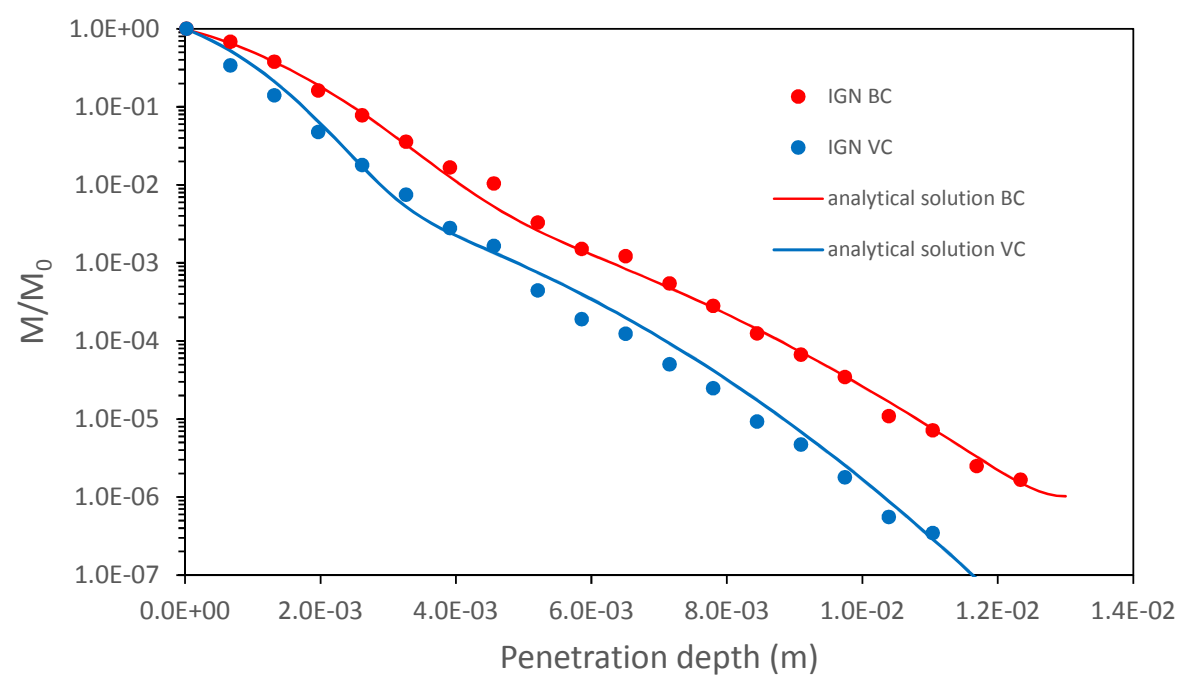

Figure 11: Total mass of cesium computed at $150 \mathrm{~h}$ for the BC (red dots) and VC (blue dots) models. The continuous lines are computed using the analytical two-parallel-pathway model. 